Supporting information

\title{
A simple way to control small-strain cavitation in die-drawn iPP
}

Dong Lyu, ${ }^{1}$ Ying Lu,,${ }^{1} *$ Glen Thompson, ${ }^{2}$ Fin Caton-Rose, ${ }^{2}$ Yuqing Lai,,${ }^{1}$ Phil Coates, ${ }^{2}$ Yongfeng Men ${ }^{1,3, *}$

${ }^{1}$ State Key Laboratory of Polymer Physics and Chemistry, Changchun Institute of Applied Chemistry, Chinese Academy of Sciences, University of Chinese Academy of Sciences, Renmin Street 5625, Changchun 130022, P. R. China

${ }^{2}$ Polymer Interdisciplinary Research Centre, University of Bradford, Bradford BD7 1DP, UK

${ }^{3}$ University of Science and Technology of China, Hefei 230026, P. R. China

\section{Corresponding Authors}

*E-mail: luying@ciac.ac.cn.

*E-mail:men@ciac.ac.cn. 


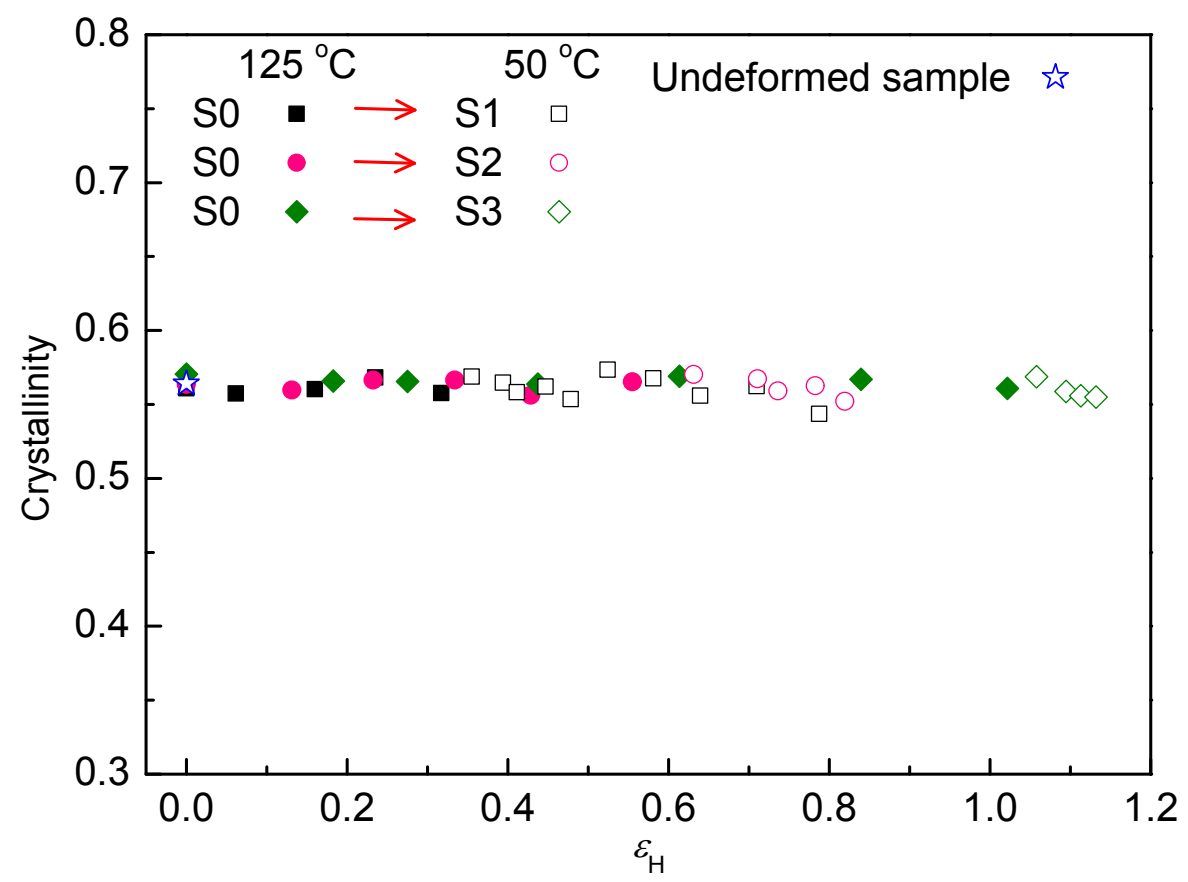

Figure S1. The crystallinity evolution of samples at different deformation stage. The undeformed sample represented the sample before the die-drawing process.

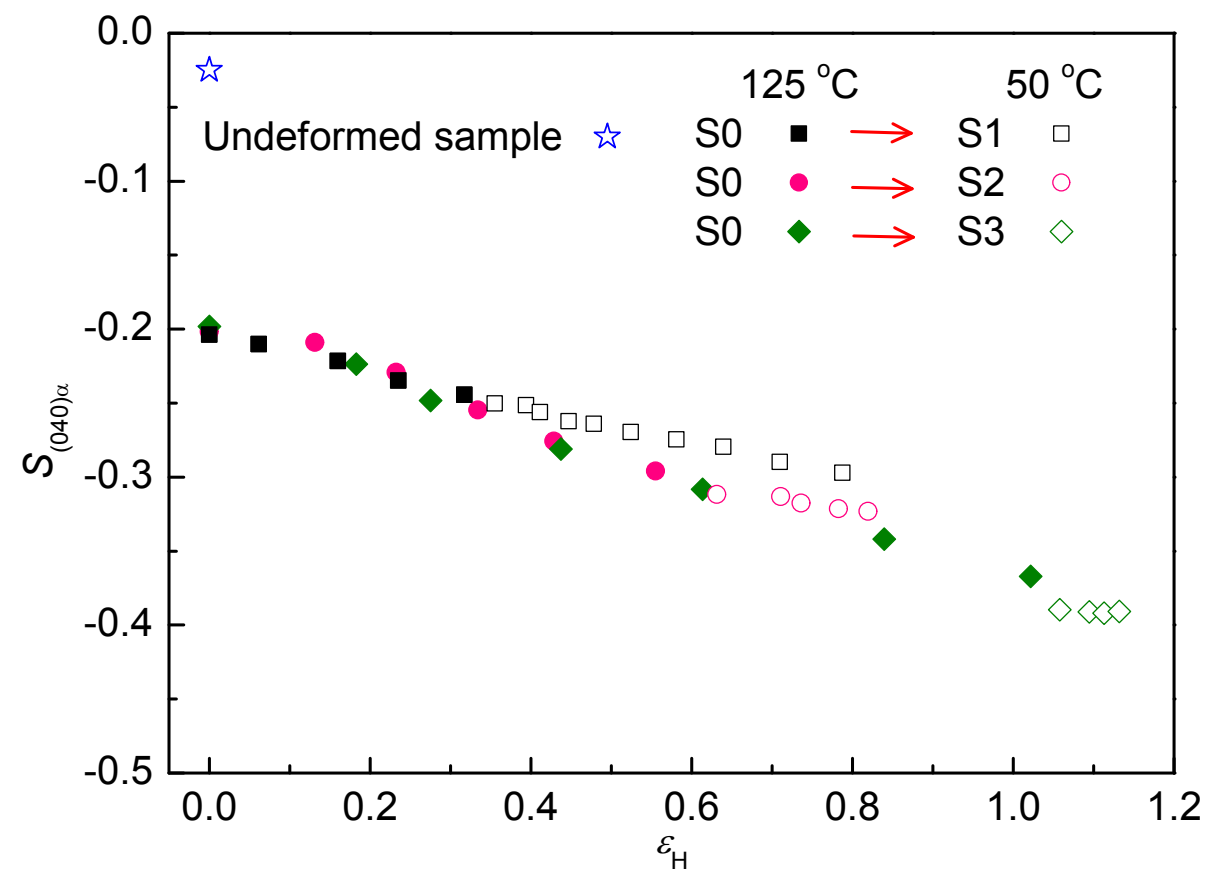


Figure S2. The orientation degree of molecular chains in the $(040)_{\alpha}$-lattice plane, $S_{(040) \alpha}$, of samples at different deformation stage. The undeformed sample represented the sample before the die-drawing process.

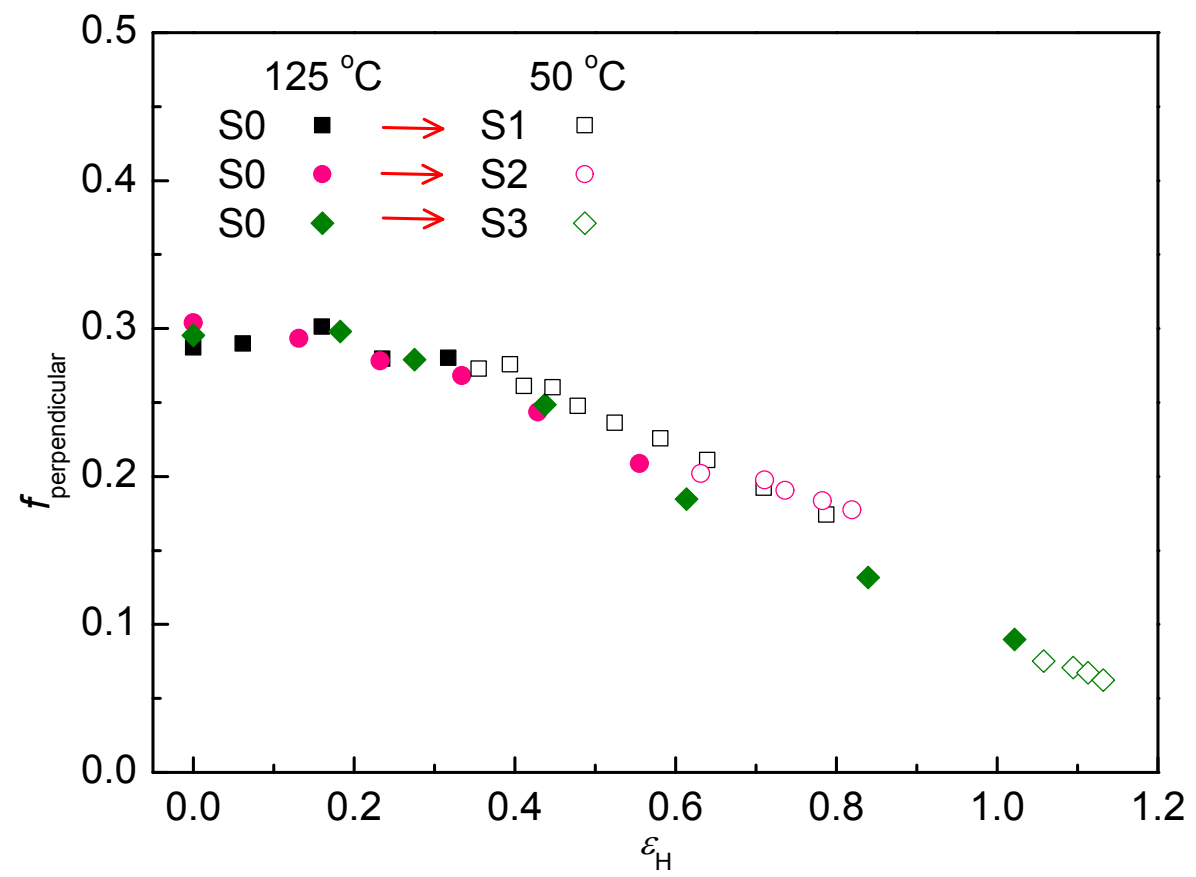

Figure S3. The fraction of perpendicular lamellae, $f_{\text {perpendicular, }}$ of different samples at different deformation stage.. 

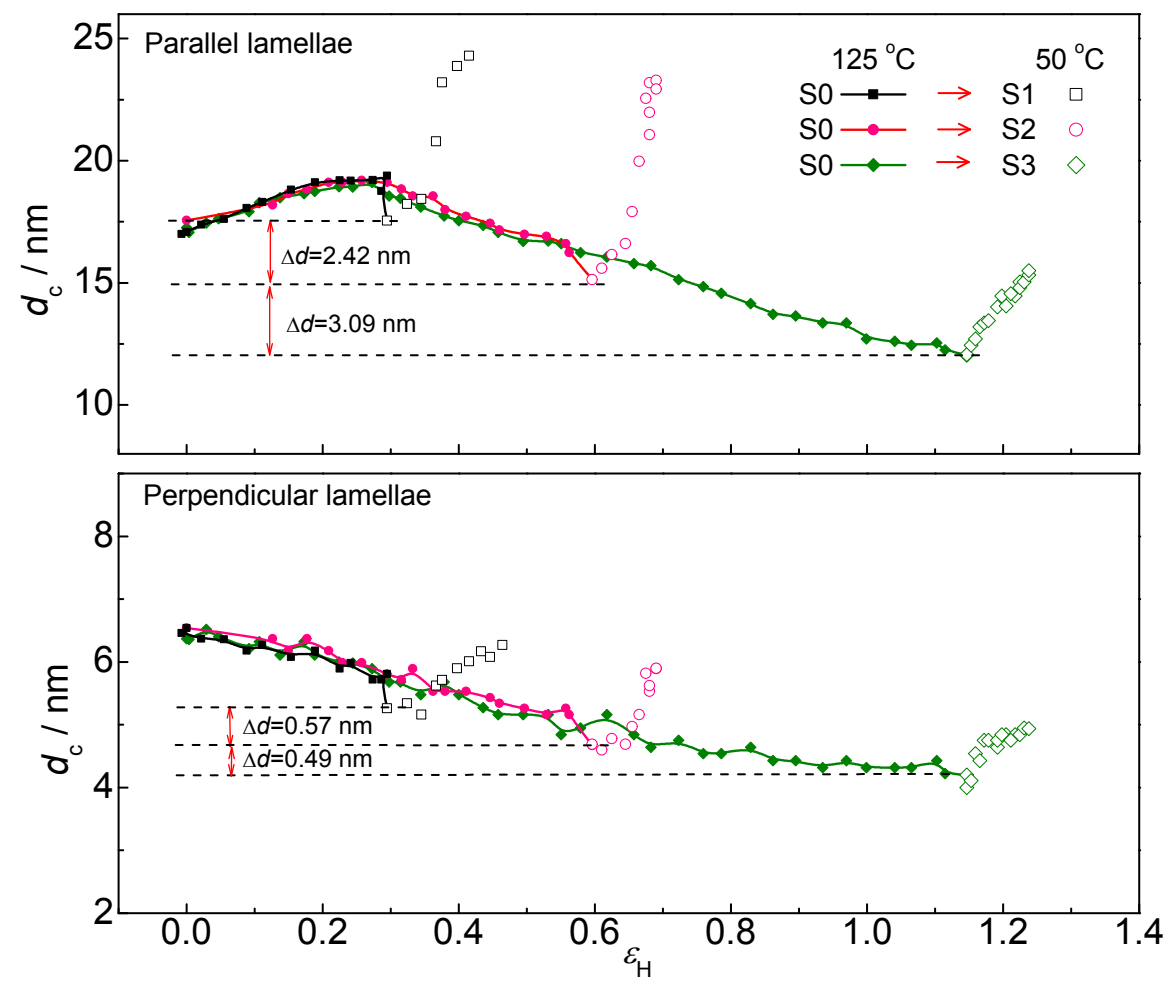

Figure S4. The evolution of lamellar thickness, $d_{c}$, of lamellae along different directions in different deformation cases.

The crystallinity of the system is about $55 \%$. During further stretching, the perpendicular lamellae were destroyed and new lamellae were recrystallized with normal along the stretching direction. Hence, the crystallinity of the parallel lamellae should be larger than $50 \%$. However, the crystallinity of the whole sample did not change a lot which indicated the crystallinity of the perpendicular lamellae decreased. Considering the ratio between perpendicular lamellae and the parallel lamellae, the crystallinity of the perpendicular lamellae was highly likely to be smaller than $50 \%$. Thus, when evaluation the perpendicular lamellae via 1D electron density correlation function analysis, the smaller values obtained were chosen to represent the thickness of 
crystalline layer. In the case of the parallel lamellae, the larger values were chosen to represent the thickness of crystalline layer.

Table S1. The thickness of crystalline layer, $d_{c}$, of different samples before and after modification at $125^{\circ} \mathrm{C}$.

\begin{tabular}{|c|c|c|c|c|c|c|}
\hline & \multicolumn{3}{|c|}{$d_{\mathrm{c}}$ of parallel lamellae } & \multicolumn{3}{c|}{$d_{\mathrm{c}}$ of perpendicular lamellae } \\
& Initial & Modified & Ratio & Initial & Modified & Ratio \\
\hline S1 & $17.08 \mathrm{~nm}$ & $17.55 \mathrm{~nm}$ & 1.03 & $6.54 \mathrm{~nm}$ & $5.26 \mathrm{~nm}$ & 0.80 \\
\hline S2 & $17.56 \mathrm{~nm}$ & $15.13 \mathrm{~nm}$ & 0.86 & $6.54 \mathrm{~nm}$ & $4.69 \mathrm{~nm}$ & 0.72 \\
\hline S3 & $17.26 \mathrm{~nm}$ & $12.04 \mathrm{~nm}$ & 0.70 & $6.37 \mathrm{~nm}$ & $4.20 \mathrm{~nm}$ & 0.66 \\
\hline
\end{tabular}

